\title{
Effects of Quality Nursing on Pulmonary Function and Quality of Life in Elderly Patients with Chronic Obstructive Pulmonary Disease
}

\author{
Shujing Zhang, Dandan Yang \\ Luohe Medical College, Luohe, Henan, China
}

Keywords: quality care; chronic obstructive pulmonary disease; pulmonary function; quality of life

\begin{abstract}
:
Aim: To investigate the clinical effect and value of quality nursing on lung function and quality of life in elderly COPD patients.

Methods: A total of 80 elderly COPD patients admitted to our hospital from March 2015 to March 2017 were randomly selected and divided into two groups $(n=40)$. The control group received routine nursing interventions, the treatment group using high-quality care, compared with two groups after the clinical effect of nursing.

Results: The FEV1, FEV1 / FVC and FEV1 scores in the control group were $(1.37 \pm 0.41),(48.06 \pm$ $5.77)$ and $(51.84 \pm 7.84)$ respectively lower than those in the treatment group $(2.57 \pm 0.39$ and 68.15 $\pm 5.61),(67.87 \pm 7.71)$. In the evaluation table of the quality of life of the treatment group, the patients' psychological function, emotional function and social function score were also significantly better than those of the control group. The difference between the two groups was significant and statistically significant $(\mathrm{P}<0.05)$

Conclusion: The implementation of quality care for elderly patients with COPD can effectively improve lung function, improve quality of life, reduce mortality and ensure the recovery of patients' health.
\end{abstract}

\section{Introduction}

Chronic obstructive pulmonary disease is a high incidence of clinical respiratory disease, as the disease continues to worsen, the patient's lung function will be a progressive development and change, if not treated will endanger the safety of life and reduce the quality of life of patients [1]. The expert study, the treatment of patients with chronic obstructive pulmonary disease during the implementation of quality care is of great significance, it can improve their prognosis and improve patient quality of life. The hospital from March 2015 to March 2017 admitted 80 elderly patients with COPD study to explore the quality of care of elderly patients with COPD nursing care, the specific report is as follows.

\section{Data and Methods}

\subsection{General File}

A total of 80 elderly COPD patients admitted to our hospital from March 2015 to March 2017 were randomly selected and divided into two groups of 40 patients with different types of nursing care . The control group received routine nursing care . Among them , male patients 21 cases and 19 female patients . The average age were $(66.37 \pm 2.17)$ years old, the year of disease was from 5 to 27 years and the average age were $(16.75 \pm 4.81)$ years. The treatment group was treated with high-quality nursing. Among them , 17 were male patients and 23 were female patients . The mean age were $64-79$ years (mean age was $65.11 \pm 3.41$ years ). The year of disease was 7 to 32 years old with an average age of $18.15 \pm 3.44$ years . The general data of two groups of patients, including age, gender, year of disease and other differences were not significant, not statistically significant, comparable $(\mathrm{P}>0.05)$.

\subsection{Method}

The control group was treated with routine nursing interventions, and nursing interventions were 
given to patients after admission on the basis of respiratory medicine. The treatment group based on routine care to implement quality care, the specific process includes: (1) medication care, in the treatment of COPD, drug treatment is the most important treatment methods, nurses should explain in detail the purpose of the patient medication, the importance of enhancing their drug compliance. After the observation of the medication situation, strictly follow the doctor's advice for the patient on time by the amount of medication, and guide patients with medication methods to ensure the treatment effect [2]. (2) diet care, nurses should be based on the patient's physical condition to develop a reasonable diet program, given to patients with high calorie, high protein, digestible, low salt and nutrient-rich foods, edema, oliguria patients need to control water and sodium intake. Pay attention to whether there will be coughing and other symptoms when eating, to avoid accidents, while exhorting patients to quit smoking, alcohol, do not eat spicy food.(3) psychological care, nurses should take the initiative to communicate more with patients, psychological comfort, to help patients understand the pathogenesis of the disease, encourage patients and build confidence. At the same time in daily care, patients can cultivate the fun of life and ask family members to accompany more, fundamentally eliminat fear, anxiety and other negative emotions. (4) Preventive care, told patients to change clothes properly when the weather changes, pay attention to warm and cold, prevent respiratory infections; improve environmental hygiene to avoid the impact of dust and irritating gases on the respiratory tract [3]. (5) rehabilitation nursing, nursing staff based on patients with cardiopulmonary function, encourage more rehabilitation exercise, such as doing Tai Chi, jogging, fast walking to improve their immunity. Reducing lip breathing and abdominal breathing exercises daily. To educate patients and their families to judge the performance of dyspnea and to arrange working hours and rests reasonably.

\subsection{Observation Index}

Compare the two groups of patients after nursing recovery of lung function. The quality of life was scored using the SF-36 scale to evaluate three aspects of the patient's psychological function, emotional function, and social function. The score was 100 and the score was directly proportional to the quality of life.

\subsection{Statistical Methods}

Application SPSS16.0 statistical software for data analysis, t-test data comparison, $\mathrm{P}<0.05$ for the difference, with statistical significance.

\section{Effects}

\subsection{Pulmonary Function Improvement}

The FEV1 , FEV1 / FVC and FEV1 scores in the control group were $(1.37 \pm 0.41),(48.06 \pm 5.77)$ and (51.84 \pm 7.84$)$ respectively lowers than those in the treatment group $(2.57 \pm 0.39)$ and $(68.15 \pm$ $5.61),(67.87 \pm 7.71)$. The difference between the two groups was significant, with statistical significance $(\mathrm{P}<0.05)$. It can be seen from Table 1 for details .

\begin{tabular}{ccccc}
\multicolumn{5}{c}{ Table 1 two groups of patients with pulmonary function recovery } \\
\hline Group & $\mathrm{n}$ & $\mathrm{FEV}_{1}$ & $\mathrm{FEV}_{1} / \mathrm{FVC}$ & $\mathrm{FEV}_{1}$ \\
\hline $\begin{array}{c}\text { Control group } \\
\text { Observation }\end{array}$ & 40 & $1.37 \pm 0.41$ & $48.06 \pm 5.77$ & $51.84 \pm 7.84$ \\
group & 40 & $2.57 \pm 0.39$ & $68.15 \pm 5.61$ & $67.87 \pm 7.71$ \\
$\mathrm{P}$ & $<0.05$ & $<0.05$ & $<0.05$ & $<0.05$ \\
\hline
\end{tabular}

\subsection{Quality of life evaluation}

The scores of psychological function, emotional function and social function of the treatment group were $(90.81 \pm 5.12),(92.16 \pm 4.81)$ and $(87.88 \pm 6.94)$ respectively, which were significantly better than those of the control group $(72.63 \pm 5.47),(77.43 \pm 5.26),(72.33 \pm 6.51)$. The difference between the two groups was significant, with statistical significance $(\mathrm{P}<0.05)$. It can be seen in 
Table 2 for details.

Table 2 evaluation form of two groups of patients quality of life

\begin{tabular}{ccccc}
\hline Group & $\mathrm{n}$ & Psychological function & $\begin{array}{c}\text { Emotional } \\
\text { function }\end{array}$ & Social function \\
\hline $\begin{array}{c}\text { Control } \\
\text { group }\end{array}$ & 40 & $72.63 \pm 5.47$ & $77.43 \pm 5.26$ & $72.33 \pm 6.51$ \\
$\begin{array}{c}\text { Observation } \\
\text { group }\end{array}$ & 40 & $90.81 \pm 5.12$ & $92.16 \pm 4.81$ & $87.88 \pm 6.94$ \\
$\mathrm{P}$ & $<0.05$ & $<0.05$ & $<0.05$ & $<0.05$ \\
\hline
\end{tabular}

\section{Conclusion}

Elderly patients are high-risk COPD patients, with the gradually deteriorating of lung function, causing obstructive ventilatory disorders, and even hypoxia and other serious acute complications, seriously affecting the patient's physical and mental health and quality of life, and increased to some extent Social burden [4]. At present, although the clinical onset of acute exacerbation of patients can be controlled, but because of deteriorating lung function, the risk of recurrence will continue to increase, there is no targeted treatment. The expert study, the treatment of COPD patients at the same time the implementation of quality care, can promote the recovery of lung function, improve patient quality of life.

In summary, the hospital treatment group has implemented high-quality nursing intervention, compared with the control group routine care, treatment group patients with lung function recovery and quality of life was significantly better than the control group [5]. Nurses according to different patient situations, from psychological to diet in many aspects of nursing, effectively improve the treatment compliance, eliminate the occurrence of adverse emotions in patients. Therefore, chronic obstructive pulmonary disease in elderly patients with quality care can promote the recovery of lung function and improve the quality of life, it is worth to clinically promote and use.

\section{References}

[1] Gu Shaoxia. Quality nursing on elderly patients with chronic obstructive pulmonary function and quality of life impact analysis [J] International Journal of Nursing, 2015, (6): 746-748.

[2] Jia Wenxiu, Wang Xiaojie, Feng Yan et al. Quality nursing on elderly patients with chronic obstructive pulmonary function and quality of life impact analysis [J]. Everyone's health (mid version), 2017,11 (6): 265.

[3] Yao Yunlan. Quality nursing on elderly patients with chronic obstructive pulmonary function and quality of life [J]. China Health Nutrition, 2017,27 (4): 207-208.

[4] Yan Xiuli. Quality nursing on elderly patients with chronic obstructive pulmonary function and quality of life [J]. Chinese Medical Guide, 2017,15 (13): 207-208.

[5] Ren Cuixia. Quality nursing on elderly patients with chronic obstructive pulmonary function and quality of life impact [J]. Everyone's health (mid version), 2017,11 (2): 256-256,257. 